\title{
Stability and Oscillation for a Coupled Hamiltonian Duffing Oscillator Model with Delays
}

\author{
Chunhua Feng ${ }^{1}$ \\ ${ }^{1}$ Department of Mathematics and Computer Science, Alabama State University, USA \\ Correspondence: Chunhua Feng, Department of Mathematics and Computer Science, Alabama State University, Mont- \\ gomery, AL, 36104, USA. E-mail: cfeng@alasu.edu
}

Received: January 2, 2018 Accepted: February 1, 2018 Online Published: February 19, 2018

doi:10.5539/jmr.v10n2p55 URL: https://doi.org/10.5539/jmr.v10n2p55

\begin{abstract}
This paper deals with a coupled Hamiltonian Duffing oscillator model with delays. The stability of the equilibrium for the system is investigated. Synchronization phenomenon occurred. Some sufficient conditions to guarantee the stability and oscillation of the trivial solution are derived. Computer simulations are provided to verify our theoretical results.
\end{abstract}

Keywords: coupled Hamiltonian Duffing oscillator model, delay, stability, oscillation

Mathematics Subject Classification: 34K11

\section{Introduction}

It is well known that various van der Pol equations, Duffing equations and van der Pol-Duffing equations describe many kinds of nonlinear oscillatory systems in physics, mechanics and engineering. They have been studied extensively in the literature (Hirano, \& Rybicki, 2003; Rompala, Rand, \& Howland, 2007; Nayfeh, \& Mook, 1979; Mickens, 1996; Brzeski, Perlikowski, \& Kapitaniak, 2014; Akhmet, \& Fen, 2012; Camargo, banez, Guerra, \& Moctezuma, 2004; Sabarathinam, \& Thamilmaran, 2015; Yalim, Welfert, \& Lopez, 2017; Pisarchik, Reategui, Salazar, Lopez, \& Boccaletti, 2006). A coupled system of simple oscillators may often exhibit many interesting phenomena different from their behavior in isolation. In 2003, Hirano and Rybicki have investigated the following van der Pol equation:

$$
\left\{\begin{array}{l}
u_{1}^{\prime \prime}+\varepsilon_{1}\left(u_{1}^{2}-1\right) u_{1}^{\prime}+u_{1}+c_{2} u_{2}=0 \\
u_{2}^{\prime \prime}+\varepsilon_{2}\left(u_{2}^{2}-1\right) u_{2}^{\prime}+u_{2}+c_{1} u_{1}=0
\end{array}\right.
$$

By using the $S^{1}$-degree theory, the authors have discussed the existence of limit cycles of system (1). Hirano and Rybicki also claimed that their results for the following coupled van der Pol equations still hold:

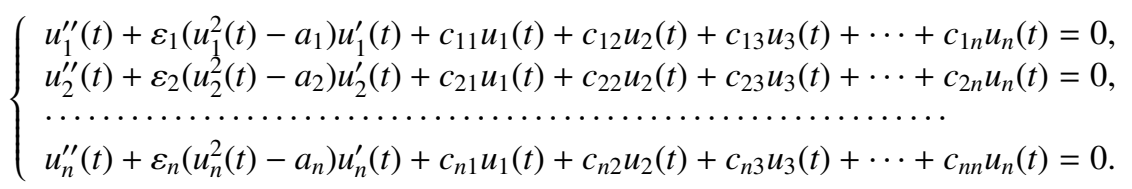

where $0<a_{i}, 0<\varepsilon_{i} \ll 1, c_{i j} \in R, 1 \leq i, j \leq n$. By means of the homotopy analysis method, Qian and Chen (Qian, \& Chen, 2010) have considered the nonlinear coupled van der Pol-Duffing oscillators model as follows:

$$
\left\{\begin{array}{l}
x_{1}^{\prime \prime}+\varepsilon\left(x_{1}^{2}-1\right) x_{1}^{\prime}+x_{1}+\delta x_{1}^{3}=\varepsilon \mu\left(x_{3}-x_{1}\right) \\
x_{2}^{\prime \prime}+\varepsilon\left(x_{2}^{2}-1\right) x_{2}^{\prime}+x_{2}+\delta x_{2}^{3}=\varepsilon \mu\left(x_{3}-x_{2}\right) \\
x_{3}^{\prime \prime}+\varepsilon\left(x_{3}^{2}-1\right) x_{3}^{\prime}+x_{3}+\delta x_{3}^{3}=\varepsilon \mu\left(x_{1}-x_{3}\right)+\varepsilon \mu\left(x_{2}-x_{3}\right)
\end{array}\right.
$$

Under some restrictive conditions, the existence of periodic solutions for the above model (3) has been obtained. Recently, Sabarathinam and Thamilmaran (Sabarathinam, \& Thamilmaran, 2015) proposed the following coupled system of nearly conservative Hamiltonian Duffing oscillators:

$$
x_{i}^{\prime \prime}(t)+b x_{i}^{\prime}(t)+\omega x_{i}(t)+\beta x_{i}^{3}(t)=\varepsilon \sum_{j=1, j \neq i}^{n} A_{i j}\left(x_{j}(t)-x_{i}(t)\right), i=1,2, \ldots, n .
$$

where $b$ is the damping coefficient, $\omega$ is the natural oscillating frequency, $\beta$ is the strength of nonlinearity, and $\varepsilon$ is the coupling coefficient, $A_{i j}$ is an adjacency matrix ( $a_{i j}=1$ if units $i$ and $j$ are connected, and 0 otherwise). The authors have discussed the stability and transient chaos for three, four and six coupled Duffing oscillators respectively. The transient 
chaos is a very interesting phenomenon in these systems. However, the above models (1)-(4) do not involve the effect of time delays. It is known that time delay is inevitable in mechanical controlling systems and manufacturing processes, nuclear reactors, rocket motors and so on. Naturally, the Duffing equations and van der Pol Duffing equations with delay have been studied by many researchers (Niu, Shen, Yang, \& Li, 2017; Wen, Shen, Yang, \& Wang, 2017; Yang, \& Zhu, 2013; Rusinek, Weremczuk, Kecik, \& Warminski, 2014; Siewe, Tchawoua, \& Rajasekar, 2012; Wang, \& Chen, 2015; Leung, Yang, \& Zhu, 2014; Zhang, Li, \& Wang, 2013; Zhang, \& Gu, 2010). For example, Wang and Chen (Wang, \& Chen, 2015) have studied weak and non-resonant double Hopf bifurcation for an $m$ coupled van der Pol oscillator with delay as follows:

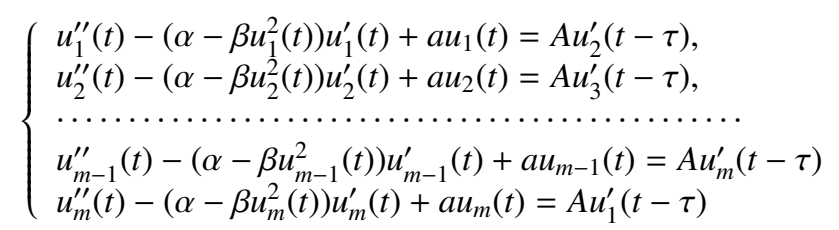

where $A$ is the coupling strength and $\tau$ is the time delay. By using the method of multiple scales, the amplitude equations are obtained. Choosing $A$ and $\tau$ as the bifurcation parameters, the dynamical behavior arising from the bifurcation is classified. Motivated by the above models, in this paper we shall discuss the following coupled time delayed Hamiltonian Duffing oscillators model:

$$
x_{i}^{\prime \prime}(t)+b_{i} x_{i}^{\prime}(t)+\omega_{i} x_{i}(t)+\beta_{i} x_{i}^{3}(t)=\varepsilon_{i} \sum_{j=1, j \neq i}^{n} A_{i j}\left(x_{j}\left(t-\tilde{\tau}_{j}\right)-x_{i}\left(t-\tilde{\tau}_{i}\right)\right), i=1,2, \ldots, n .
$$

where $b_{i}, \omega_{i}, \beta_{i}, \varepsilon_{i}$ are constants, $0<\varepsilon_{i} \ll 1$ and time delay $\tilde{\tau}_{i} \geq 0(i=1,2, \ldots, n)$. The initial condition is $x_{i}(t)=$ $\varphi_{i}(t), t \in\left[-\tilde{\tau^{*}}, 0\right](i=1,2, \ldots, n)$, where $\tilde{\tau^{*}}=\max \left\{\tilde{\tau_{1}}, \tilde{\tau_{2}}, \ldots, \tilde{\tau_{n}}\right\}$. By using the Mathematical Analysis Method, we discuss the stability of the equilibrium point and oscillatory behavior of the trivial solution. Our method is different from the Sabarathinam's approach. It was emphasized that in the model (6), the synchronization phenomenon appeared. The stability and oscillation of three, four and six coupled Hamiltonian Duffing oscillators are indeed the same as that of an uncoupled equation. It is convenient to write (6) as an equivalent $2 n$ dimensional first order system:

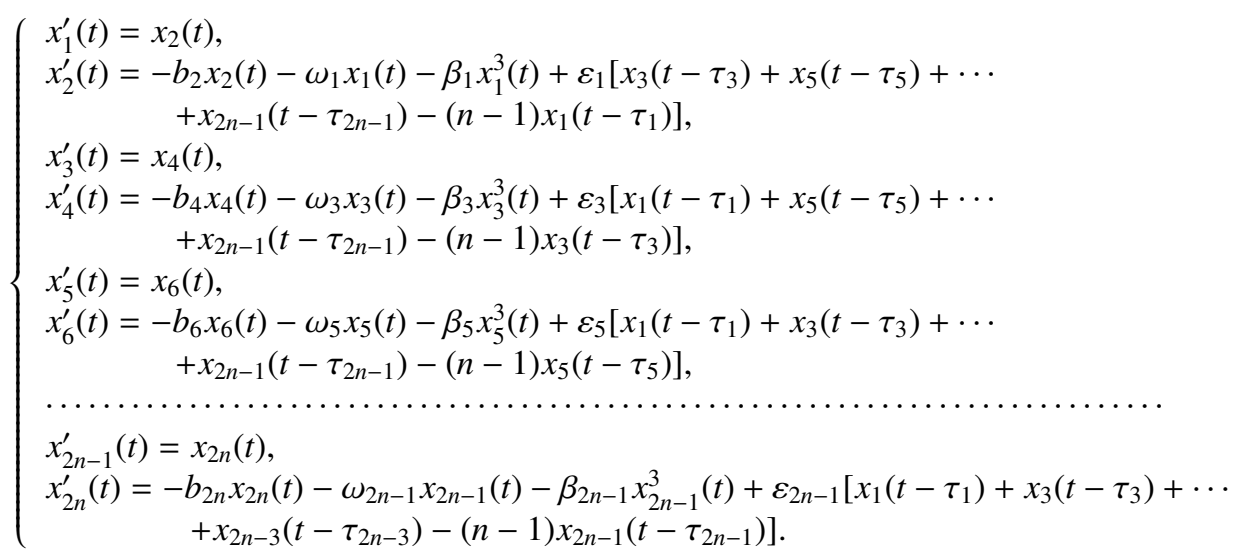

where $\tau_{2 i-1}=\tilde{\tau}_{i}(i=1,2, \ldots, n)$. The matrix form of $(7)$ is as follows:

$$
x^{\prime}(t)=P x(t)+Q x(t-\tau)+f(x(t))
$$

where $x(t)=\left[x_{1}(t), x_{2}(t), \cdots, x_{2 n}(t)\right]^{T}, x(t-\tau)=\left[x_{1}\left(t-\tau_{1}\right), 0, x_{3}\left(t-\tau_{3}\right), \cdots, x_{2 n-1}\left(t-\tau_{2 n-1}\right), 0\right]^{T}, f(x(t))$ $=\left[0,-\beta_{1} x_{1}^{3}(t), 0,-\beta_{3} x_{3}^{3}(t), 0, \cdots, 0,-\beta_{2 n-1} x_{2 n-1}^{3}(t), 0\right]^{T}$. Both $P=\left(p_{i j}\right)_{2 n \times 2 n}$ and $Q=\left(q_{i j}\right)_{2 n \times 2 n}$ are $2 n$ by $2 n$ matrices as follows:

$$
P=\left(\begin{array}{cccccccccc}
0 & 1 & 0 & 0 & 0 & 0 & \cdots & 0 & 0 & 0 \\
-\omega_{1} & -b_{2} & 0 & 0 & 0 & 0 & \cdots & 0 & 0 & 0 \\
0 & 0 & 0 & 1 & 0 & 0 & \cdots & 0 & 0 & 0 \\
0 & 0 & -\omega_{3} & -b_{4} & 0 & 0 & \cdots & 0 & 0 & 0 \\
\cdots & \cdots & \cdots & \cdots & \cdots & \cdots & \cdots & \cdots & \cdots & \cdots \\
0 & 0 & 0 & 0 & 0 & 0 & \cdots & 1 & 0 & 0 \\
0 & 0 & 0 & 0 & 0 & 0 & \cdots & -b_{2 n-2} & 0 & 0 \\
0 & 0 & 0 & 0 & 0 & 0 & \cdots & 0 & 0 & 1 \\
0 & 0 & 0 & 0 & 0 & 0 & \cdots & 0 & -\omega_{2 n-1} & -b_{2 n}
\end{array}\right),
$$




$$
Q=\left(\begin{array}{cccccccccc}
0 & 0 & 0 & 0 & 0 & 0 & \cdots & 0 & 0 & 0 \\
-(n-1) \varepsilon_{1} & 0 & \varepsilon_{1} & 0 & \varepsilon_{1} & 0 & \cdots & 0 & \varepsilon_{1} & 0 \\
0 & 0 & 0 & 0 & 0 & 0 & \cdots & 0 & 0 & 0 \\
\varepsilon_{3} & 0 & -(n-1) \varepsilon_{3} & 0 & \varepsilon_{3} & 0 & \cdots & 0 & \varepsilon_{3} & 0 \\
\cdots & \cdots & \cdots & \cdots & \cdots & \cdots & \cdots & \cdots & \cdots & \cdots \\
0 & 0 & 0 & 0 & 0 & 0 & \cdots & 0 & 0 & 0 \\
\varepsilon_{5} & 0 & \varepsilon_{5} & 0 & -(n-1) \varepsilon_{5} & 0 & \cdots & 0 & \varepsilon_{5} & 0 \\
0 & 0 & 0 & 0 & 0 & 0 & \cdots & 0 & 0 & 0 \\
\varepsilon_{2 n-1} & 0 & \varepsilon_{2 n-1} & 0 & \varepsilon_{2 n-1} & 0 & \cdots & 0 & -(n-1) \varepsilon_{2 n-1} & 0
\end{array}\right) .
$$

The linearized system of (8) is the following:

$$
x^{\prime}(t)=P x(t)+Q x(t-\tau)
$$

We adopt the following norms of vectors and matrices in this paper (Desoer, \& Vidyasagar, 1977): $\|z(t)\|=\sum_{i=1}^{n}\left|z_{i}(t)\right|,\|A\|=$ $\sum_{i=1}^{n}\left|a_{i j}\right|$, the measure $\mu(A)$ of a matrix $A$ is defined by $\mu(A)=\lim _{\theta \rightarrow 0^{+}} \frac{\|I+\theta A\|-1}{\theta}$, which for the chosen norms reduces to $\mu(A)=\max _{1 \leq j \leq n}\left[a_{j j}+\sum_{i=1, i \neq j}^{n}\left|a_{i j}\right|\right] . A>0$ (respectively, $A<0$ ) which indicates that $A$ is a positive (negative) definite matrix.

\section{Preliminaries}

Lemma 1 Suppose that $b_{i}, \omega_{i}, \beta_{i}, \varepsilon_{i}$ are constants, $0<\varepsilon_{i} \ll 1$. If the matrix $R(=P+Q)$ is a nonsingular matrix, then system (8) has a unique equilibrium point.

Proof If $x^{*}=\left[x_{1}^{*}, x_{2}^{*}, \cdots, x_{2 n}^{*}\right]^{T}$ is an equilibrium point of system (8), then $x^{*}$ is a constant solution of the following algebraic equation

$$
P x^{*}+Q x^{*}+f\left(x^{*}\right)=(P+Q) x^{*}+f\left(x^{*}\right)=R x^{*}+f\left(x^{*}\right)=\mathbf{0} .
$$

Suppose that $y^{*}=\left[y_{1}^{*}, y_{2}^{*}, \cdots, y_{2 n}^{*}\right]^{T}$ is another equilibrium point of system (8), then we have

$$
(P+Q)\left(x^{*}-y^{*}\right)+f\left(x^{*}\right)-f\left(y^{*}\right)=R\left(x^{*}-y^{*}\right)+f\left(x^{*}\right)-f\left(y^{*}\right)=\mathbf{0} .
$$

where $f\left(x^{*}\right)-f\left(y^{*}\right)=\left[0,-\beta_{1}\left(x_{1}^{* 3}-y_{1}^{* 3}\right), 0,-\beta_{3}\left(x_{3}^{* 3}-y_{3}^{* 3}\right), 0, \cdots, 0,-\beta_{2 n-1}\left(x_{2 n-1}^{* 3}-y_{2 n-1}^{* 3}\right), 0\right]^{T}=\left[0,-\beta_{1}\left(x_{1}^{* 2}+x_{1}^{*} y_{1}^{*}+\right.\right.$ $\left.\left.y_{1}^{* 2}\right)\left(x_{1}^{*}-y_{1}^{*}\right), 0,-\beta_{3}\left(x_{3}^{* 2}+x_{3}^{*} y_{3}^{*}+y_{3}^{* 2}\right)\left(x_{3}^{*}-y_{3}^{*}\right), 0, \cdots, 0,-\beta_{2 n-1}\left(x_{2 n-1}^{* 2}+x_{2 n-1}^{*} y_{2 n-1}^{*}+y_{2 n-1}^{* 2}\right)\left(x_{2 n-1}^{*}-y_{2 n-1}^{*}\right), 0\right]^{T}$. From $(11)$ we have

$$
\left[R+g\left(x^{*}\right)-g\left(y^{*}\right)\right]\left(x^{*}-y^{*}\right)=\mathbf{0} .
$$

where $g\left(x^{*}\right)-g\left(y^{*}\right)=\left[0,-\beta_{1}\left(x_{1}^{* 2}+x_{1}^{*} y_{1}^{*}+y_{1}^{* 2}\right), 0,-\beta_{3}\left(x_{3}^{* 2}+x_{3}^{*} y_{3}^{*}+y_{3}^{* 2}\right), 0, \cdots, 0\right.$, $\left.-\beta_{2 n-1}\left(x_{2 n-1}^{* 2}+x_{2 n-1}^{*} y_{2 n-1}^{*}+y_{2 n-1}^{* 2}\right), 0\right]^{T}$. Since $R$ is a nonsingular matrix, and for any values $x_{i}^{*}, y_{i}^{*}$ we always have $x_{i}^{* 2}+$ $x_{i}^{*} y_{i}^{*}+y_{i}^{* 2} \geq 0(i=1,3,5, \cdots, 2 n-1)$, and $R+g\left(x^{*}\right)-g\left(y^{*}\right) \neq \mathbf{0}$ (vector). Thus from (10) we get

$$
x^{*}-y^{*}=\mathbf{0} \text {. }
$$

This means that system (8) has a unique equilibrium point. Obviously, this equilibrium point exactly is the zero point.

Lemma 2 Suppose that $b_{i}, \omega_{i}, \beta_{i}, \varepsilon_{i}$ are positive constants, $0<\varepsilon_{i} \ll 1$. Then all solutions of system (8) are bounded if system (8) has a bounded particular solution.

Proof It is known that time delay may induce the instability of the solutions. It does not affect the boundedness of the solutions. In order to prove the boundedness of the solutions of system (8) (or equivalent system (6)), we need only consider the boundedness of system (6) in which all time delays are equal to zeros. For convenience we rewrite such without time delays system as follows:

$$
x_{i}^{\prime \prime}(t)+b_{i} x_{i}^{\prime}(t)+\omega_{i} x_{i}(t)=-\beta_{i} x_{i}^{3}(t)+\varepsilon_{i} \sum_{j=1, j \neq i}^{n} A_{i j}\left(x_{j}(t)-x_{i}(t)\right), i=1,2, \ldots, n .
$$

According to the basic theory of differential equations, the general solution of nonhomogeneous system (14) equal to the sum of the general solution of homogeneous system associated with (14) and a particular solution of system (14). The homogeneous system corresponding to (14) is the following:

$$
x_{i}^{\prime \prime}(t)+b_{i} x_{i}^{\prime}(t)+\omega_{i} x_{i}(t)=0, i=1,2, \ldots, n .
$$


Since $b_{i}, \omega_{i}$ are positive constants, the characteristic equation of (15) is as follows:

$$
\lambda^{2}+b_{i} \lambda+\omega_{i}=0, i=1,2, \ldots, n .
$$

Obviously, $\lambda_{1 i}=\frac{-b_{i}+\sqrt{b_{i}^{2}-4 \omega_{i}}}{2}, \lambda_{2 i}=\frac{-b_{i}-\sqrt{b_{i}^{2}-4 \omega_{i}}}{2}(i=1,2, \cdots, n)$ are negative numbers $\left(b_{i}^{2}-4 \omega_{i} \geq 0\right)$, or have negative real parts $\left(b_{i}^{2}-4 \omega_{i}<0\right)$. So the general solution of system (15) is bounded. Since the particular solution is bounded, hence, the solutions of system (8) are bounded.

Correspondingly in system (9), we consider two special cases:

$$
x^{\prime}(t)=P x(t)+Q x\left(t-\tau^{*}\right)
$$

where $x\left(t-\tau^{*}\right)=\left[x_{1}\left(t-\tau^{*}\right), x_{2}\left(t-\tau^{*}\right), \cdots, x_{2 n}\left(t-\tau^{*}\right)\right]^{T}, \tau^{*}=\max \left\{\tau_{1}, \tau_{3}, \cdots, \tau_{2 n-1}\right\}$. and

$$
x^{\prime}(t)=P x(t)+Q x\left(t-\tau_{*}\right)
$$

where $x\left(t-\tau_{*}\right)=\left[x_{1}\left(t-\tau_{*}\right), x_{2}\left(t-\tau_{*}\right), \cdots, x_{2 n}\left(t-\tau_{*}\right)\right]^{T}, \tau_{*}=\min \left\{\tau_{1}, \tau_{3}, \cdots, \tau_{2 n-1}\right\}$.

\section{Main Results}

Theorem 1 Assume that system (8) has a unique equilibrium point. If there exist symmetric, positive definite matrices $S_{1}$ and $S_{2}$ such that

$$
P^{T} S_{1}+S_{1} P+S_{2}+S_{1}+S_{1} Q S_{2}^{T} Q^{T} S_{1}<0
$$

then the trivial solution of system (8) is asymptotically stable.

Proof Note that $f(x)$ is high order infinitesimal as $x \rightarrow 0$. Hence we only need to consider the stability of the trivial solution of system (9). According to the theory of delayed differential equation, the asymptotic stability of the trivial solution of system (17) guarantees the asymptotic stability of the trivial solution of system (9). Therefore, in the following we consider system (17). Let $V\left(x_{t}\right)$ be a Lyaponov functional given by

$$
V\left(x_{t}\right)=x(t)^{T} S_{1} x(t)+\int_{t-\tau^{*}}^{t} x(s)^{T} S_{2} x(s) d s
$$

Calculating the upper right derivative of $V\left(x_{t}\right)$ along the solution of (17) yields

$$
\begin{aligned}
\left.D^{+} V\left(x_{t}\right)\right|_{(17)}= & x(t)^{T}\left[P^{T} S_{1}+S_{1} P+S_{2}\right] x(t)+x(t)^{T} S_{1} Q x\left(t-\tau^{*}\right) \\
& +x\left(t-\tau^{*}\right)^{T} Q^{T} S_{1} x(t)-x\left(t-\tau^{*}\right)^{T} S_{2} x\left(t-\tau^{*}\right)
\end{aligned}
$$

The right hand of the above equation is expressed below as a quadratic form. In order to ensure asymptotic stability of the system (17) this quadratic form has to be negative definite as follows:

$$
W(t)^{T} M W(t)<0
$$

where $W(t)^{T}=\left[x(t)^{T}, x\left(t-\tau^{*}\right)^{T}\right]$. Noting that $S_{1}, S_{2}$ are symmetric matrices, then

$$
M=\left(\begin{array}{cc}
P^{T} S_{1}+S_{1} P+S_{2} & S_{1} Q \\
Q^{T} S_{1} & -S_{2}
\end{array}\right)=\left(\begin{array}{cc}
P^{T} S_{1}+S_{1} P+S_{2} & S_{1} Q \\
\left(S_{1} Q\right)^{T} & -S_{2}
\end{array}\right) .
$$

Kreindler and Jameson (Kreindler, \& Jameson, 1972) pointed out that for the matrices $U_{11}, U_{12}, U_{22}$ with appropriate dimensions, the condition

$$
\left(\begin{array}{cc}
U_{11} & U_{12} \\
U_{12}^{T} & U_{22}
\end{array}\right)<0
$$

is equivalent to $U_{11}<0$ and $U_{11}-U_{12} U_{22}^{-1} U_{12}^{T}<0$. Applying this fact to condition (21), we obtain

$$
\begin{gathered}
P^{T} S_{1}+S_{1} P+S_{2}<0 \\
P^{T} S_{1}+S_{1} P+S_{2}+S_{1}+S_{1} Q S_{2}^{T} Q^{T} S_{1}<0
\end{gathered}
$$

Only the latter relation is relevant, since it includes condition (22). Therefore, $\left.D^{+} V\left(x_{t}\right)\right|_{(17)}<0$ if condition (19) is satisfied. This completes the proof. 
Theorem 2 Assume that system (8) has a unique equilibrium point. Let the eigenvalues of matrix $P$ be $\rho_{i}(i=1,2, \ldots, 2 n)$. If $\operatorname{Re}\left(\rho_{i}\right) \leq-a<0(i=1,2, \ldots, 2 n)$, then the unique equilibrium point of system (8) is asymptotically stable.

Proof Note that each eigenvalue of matrix $Q$ is zero. Since $\operatorname{Re}\left(\rho_{i}\right) \leq-a<0(i=1,2, \ldots, 2 n)$, there exists $L \geq 1$ such that $\left\|e^{(P+Q) t}\right\| \leq L e^{-a t}$. Similar to Theorem 1, we still consider the asymptotic stability of the trivial solution of system (17). Rewrite (17) in the form

$$
\begin{aligned}
x^{\prime}(t) & =(P+Q) x(t)-Q \int_{t-\tau^{*}}^{t} x^{\prime}(s) d s \\
& =(P+Q) x(t)-Q \int_{t-\tau^{*}}^{t}\left(P x(s)+Q x\left(s-\tau^{*}\right)\right) d s, t \geq \tau^{*}
\end{aligned}
$$

By variation of parameter, this leads to

$$
x(t)=e^{(P+Q)\left(t-\tau^{*}\right)} x\left(\tau^{*}\right)-\int_{\tau^{*}}^{t} d s \int_{s-\tau^{*}}^{s} e^{(P+Q)(t-s)} Q\left(P x(u)+Q x\left(u-\tau^{*}\right)\right) d u, t \geq \tau^{*}
$$

and hence for $t \geq \tau^{*}$, we have

$$
\|x(t)\| \leq A L e^{-a\left(t-\tau^{*}\right)}+L\|Q\| \int_{\tau^{*}}^{t} d s \int_{s-\tau^{*}}^{s} e^{-a(t-s)}\left(\|P\|\|x(u)\|+\|Q\|\left\|x\left(u-\tau^{*}\right)\right\|\right) d u, t \geq \tau^{*}
$$

where $A=\sup _{t \in\left[-\tau^{*}, \tau^{*}\right]}\|x(t)\|$. For $t \geq \tau^{*}$ define

$$
\|y(t)\|=A L e^{-a\left(t-\tau^{*}\right)}+L\|Q\| \int_{\tau^{*}}^{t} d s \int_{s-\tau^{*}}^{s} e^{-a(t-s)}\left(\|P\|\|y(u)\|+\|Q\|\left\|y\left(u-\tau^{*}\right)\right\|\right) d u, t \geq \tau^{*}
$$

According to the comparison theorem of differential equations, we have $|x(t)| \leq|y(t)|$. We will show next that there exists a positive constant $b(b<a)$ such that $y(t)$ can be written as the form

$$
y(t)=A L e^{-b\left(t-\tau^{*}\right)}, t \geq \tau^{*}
$$

Indeed,

$$
\begin{aligned}
& A L e^{-a\left(t-\tau^{*}\right)}+L\|Q\| \int_{\tau^{*}}^{t} d s \int_{s-\tau^{*}}^{s} e^{-a(t-s)}\left(\|P\|\|y(u)\|+\|Q\|\left\|y\left(u-\tau^{*}\right)\right\|\right) d u \\
= & A L e^{-a\left(t-\tau^{*}\right)}+L\|Q\| \int_{\tau^{*}}^{t} d s \int_{s-\tau^{*}}^{s} e^{-a(t-s)}\left(\|P\| A L e^{-b\left(u-\tau^{*}\right)}+\|Q\| A L e^{-b\left(u-2 \tau^{*}\right)}\right) d u \\
= & A L e^{-a\left(t-\tau^{*}\right)}+L\|Q\| \frac{A L\left(\|P\| e^{b \tau^{*}}+\|Q\| e^{2 b \tau^{*}}\right)}{-b} \int_{\tau^{*}}^{t} e^{-a(t-s)}\left(e^{-b s}-e^{-b\left(s-\tau^{*}\right)}\right) d s \\
= & A L e^{-a\left(t-\tau^{*}\right)}+\frac{A L^{2}\|Q\|\left(\|P\| e^{b \tau^{*}}+\|Q\| e^{2 b \tau^{*}}\right)}{-b} e^{-a t}\left(1-e^{b \tau^{*}}\right) \int_{\tau^{*}}^{t} e^{(a-b) s} d s \\
= & A L e^{-a\left(t-\tau^{*}\right)}+\frac{A L^{2}\|Q\|\left(\|P\|+\|Q\| e^{b \tau^{*}}\right)\left(e^{b \tau^{*}}-1\right)}{b(a-b)} e^{-a t} e^{b \tau^{*}}\left(e^{(a-b) t}-e^{(a-b) \tau^{*}}\right) \\
= & A L e^{-a\left(t-\tau^{*}\right)}+\frac{A L^{2}\|Q\|\left(\|P\|+\|Q\| e^{b \tau^{*}}\right)\left(e^{b \tau^{*}}-1\right)}{b(a-b)}\left(e^{-b\left(t-\tau^{*}\right)}-e^{-a\left(t-\tau^{*}\right)}\right)
\end{aligned}
$$

Select the positive constant $b(b<a)$ such that $\frac{L\|Q\|\left(\|P\|+\|Q\| e^{b \tau^{*}}\right)\left(e^{b \tau^{*}}-1\right)}{b(a-b)}=1$, then

$$
\begin{aligned}
& A L e^{-a\left(t-\tau^{*}\right)}+\frac{A L^{2}\|Q\|\left(\|P\|+\|Q\| e^{b \tau^{*}}\right)\left(e^{b \tau^{*}}-1\right)}{b(a-b)}\left(e^{-b\left(t-\tau^{*}\right)}-e^{-a\left(t-\tau^{*}\right)}\right) \\
= & A L e^{-a\left(t-\tau^{*}\right)}+A L \frac{L\|Q\|\left(\|P\|+\|Q\| b e^{*}\right)\left(e^{b \tau^{*}}-1\right)}{b(a-b)}\left(e^{-b\left(t-\tau^{*}\right)}-e^{-a\left(t-\tau^{*}\right)}\right) \\
= & A L e^{-a\left(t-\tau^{*}\right)}+A L\left(e^{-b\left(t-\tau^{*}\right)}-e^{-a\left(t-\tau^{*}\right)}\right) \\
= & A L e^{-b\left(t-\tau^{*}\right)}=y(t)
\end{aligned}
$$

holds. From (29), we have $y(t) \rightarrow 0$ as $t \rightarrow \infty$, implying that $x(t) \rightarrow 0$ as $t \rightarrow \infty$, and the proof is completed.

Theorem 3 Assume that system (8) has a unique equilibrium point. If there exists at least one eigenvalue of matrix $P$ with $\operatorname{Re}\left(\rho_{j}\right)>0$, then the trivial solution of system (8) is unstable. System (8) generates a permanent oscillatory solution. 
Proof Since $f(x)$ is high order infinitesimal as $x \rightarrow 0$, the instability of the trivial solution of system (9) implies the instability of the trivial solution of system (8). According to the theory of delayed differential equation, the trivial solution of system (8) is unstable if and only if the trivial solution of system (18) is unstable. So we only consider system (18). Note that each characteristic value of matrix $Q$ is zero. Hence the characteristic equation of system (18) is as follows

$$
\operatorname{det}\left(\lambda I-P-Q e^{-\lambda \tau_{*}}\right)=\operatorname{det}(\lambda I-P)=\prod_{i=1}^{2 n}\left(\lambda_{i}-\rho_{i}\right)=0
$$

where $I$ is the $2 n$ by $2 n$ identity matrix. By the assumption, there is at least one $j$ such that $\operatorname{Re}\left(\lambda_{j}\right)=\operatorname{Re}\left(\rho_{j}\right)>0$. This means that the trivial solution (18) is unstable, implying that the trivial solution of (8) is unstable. If system (8) has a unique equilibrium point, that guarantees system (18) has a unique equilibrium point. The instability of the trivial solution with uniqueness of the equilibrium point will force system (18)(thus system (8)) to generate a permanent oscillatory solution. The proof is completed.

Theorem 4 Assume that system (8) has a unique equilibrium point. If the real part of each eigenvalue of the matrix $P$ is zero, then the trivial solution of system (8) is unstable, implying that system (8) generates a permanent oscillatory solution.

Proof Similar to theorem 3, we only consider system (18). Since the real part of each eigenvalue of the matrix $P$ is zero, from (32), this means that the eigenvalues of system (18) are pure imaginary numbers $\lambda_{k}=i \omega_{k}(k=1,2, \cdots, 2 n)$. Noting that the trigonometric functions $\cos \omega_{k} t, \sin \omega_{k} t$ are not convergent to zero, this implies that the trivial solution of system (18) is unstable. The instability of the trivial solution with uniqueness of the equilibrium point will force system (18), hence system (8) to generate a permanent oscillatory solution. The proof is completed.

\section{Simulation Results}

Example 1. First we consider case of $n=3$.

$$
\left\{\begin{array}{l}
x_{1}^{\prime}(t)=x_{2}(t), \\
x_{2}^{\prime}(t)=-b_{2} x_{2}(t)-\omega_{1} x_{1}(t)-\beta_{1} x_{1}^{3}(t)+\varepsilon_{1}\left[x_{3}\left(t-\tau_{3}\right)+x_{5}\left(t-\tau_{5}\right)-2 x_{1}\left(t-\tau_{1}\right)\right], \\
x_{3}^{\prime}(t)=x_{4}(t), \\
x_{4}^{\prime}(t)=-b_{4} x_{4}(t)-\omega_{3} x_{3}(t)-\beta_{3} x_{3}^{3}(t)+\varepsilon_{3}\left[x_{1}\left(t-\tau_{1}\right)+x_{5}\left(t-\tau_{5}\right)-2 x_{3}\left(t-\tau_{3}\right)\right], \\
x_{5}^{\prime}(t)=x_{6}(t), \\
x_{6}^{\prime}(t)=-b_{6} x_{6}(t)-\omega_{5} x_{5}(t)-\beta_{5} x_{5}^{3}(t)+\varepsilon_{5}\left[x_{1}\left(t-\tau_{1}\right)+x_{3}\left(t-\tau_{3}\right)-2 x_{5}\left(t-\tau_{5}\right)\right] .
\end{array}\right.
$$

We fixed $b_{2}=0.25, b_{4}=0.35, b_{6}=0.55 ; \omega_{1}=1.2, \omega_{3}=1.5, \omega_{5}=1.8 ; \varepsilon_{1}=0.0005, \varepsilon_{3}=0.0008, \varepsilon_{5}=0.0006 ; \beta_{1}=$ $0.25, \beta_{2}=0.2, \beta_{3}=0.3 ; \tau_{1}=12, \tau_{3}=10, \tau_{5}=14$, respectively. The eigenvalues of matrix $P$ are $-0.1250 \pm 1.0883 i,-0.1750 \pm$ $1.2122 i,-0.2750 \pm 1.3132 i$. The real part of each eigenvalue of matrix $P$ is negative, the trivial solution is convergent according to Theorem 2 (see Fig. 1 ). When we fixed $b_{2}=-0.00025, b_{4}=0.0035, b_{6}=-0.00015 ; \omega_{1}=0.12, \omega_{3}=$ $0.15, \omega_{5}=0.18$, and other parameters are the same as Fig. 1 . The eigenvalues of matrix $P$ are $0.0001 \pm 0.3464 i,-0.0018 \pm$ $0.3873 i, 0.0001 \pm 0.4243 i$, respectively. Noting that there exist four eigenvalues of matrix $P$ that are positive real parts. Based on Theorem 3, system (33) generates an oscillatory solution (see Fig.2).

Example 2. We then consider case of $n=4$.

$$
\left\{\begin{array}{l}
x_{1}^{\prime}(t)=x_{2}(t) \\
x_{2}^{\prime}(t)=-b_{2} x_{2}(t)-\omega_{1} x_{1}(t)-\beta_{1} x_{1}^{3}(t)+\varepsilon_{1}\left[x_{3}\left(t-\tau_{3}\right)+x_{5}\left(t-\tau_{5}\right)+x_{7}\left(t-\tau_{7}\right)-3 x_{1}\left(t-\tau_{1}\right)\right], \\
x_{3}^{\prime}(t)=x_{4}(t) \\
x_{4}^{\prime}(t)=-b_{4} x_{4}(t)-\omega_{3} x_{3}(t)-\beta_{3} x_{3}^{3}(t)+\varepsilon_{3}\left[x_{1}\left(t-\tau_{1}\right)+x_{5}\left(t-\tau_{5}\right)+x_{7}\left(t-\tau_{7}\right)-3 x_{3}\left(t-\tau_{3}\right)\right], \\
x_{5}^{\prime}(t)=x_{6}(t) \\
x_{6}^{\prime}(t)=-b_{6} x_{6}(t)-\omega_{5} x_{5}(t)-\beta_{5} x_{5}^{3}(t)+\varepsilon_{5}\left[x_{1}\left(t-\tau_{1}\right)+x_{3}\left(t-\tau_{3}\right)+x_{7}\left(t-\tau_{7}\right)-3 x_{5}\left(t-\tau_{5}\right)\right], \\
x_{7}^{\prime}(t)=x_{8}(t) \\
x_{8}^{\prime}(t)=-b_{8} x_{8}(t)-\omega_{7} x_{7}(t)-\beta_{7} x_{7}^{3}(t)+\varepsilon_{7}\left[x_{1}\left(t-\tau_{1}\right)+x_{3}\left(t-\tau_{3}\right)+x_{5}\left(t-\tau_{5}\right)-3 x_{7}\left(t-\tau_{7}\right)\right] .
\end{array}\right.
$$

We select the values of parameters as: $b_{2}=0.65, b_{4}=0.25, b_{6}=0.45, b_{8}=0.28 ; \omega_{1}=0.20, \omega_{3}=0.16, \omega_{5}=0.18, \omega_{7}=$ $0.08 ; \varepsilon_{1}=0.0012, \varepsilon_{3}=0.0015, \varepsilon_{5}=0.0016, \varepsilon_{7}=0.0018 ; \beta_{1}=0.012, \beta_{3}=0.15, \beta_{5}=0.16, \beta_{7}=0.18 ; \tau_{1}=12, \tau_{3}=$ $10, \tau_{5}=14, \tau_{7}=15$, respectively. The eigenvalues of matrix $P$ are $-0.3250 \pm 0.3072 i,-0.1250 \pm 0.3800 i,-0.2250 \pm$ $0.3597 i,-0.1400 \pm 0.2458 i$, respectively. Since the real part of each eigenvalue is negative, the trivial solution is convergent according to Theorem 2 (see Fig.3). However, when we changed $b_{2}=-0.00065, b_{4}=0.00025, b_{6}=-0.00045, b_{8}=$ $0.00028 ; \omega_{1}=4.2, \omega_{3}=3.5, \omega_{5}=2.4, \omega_{7}=5.5$, the other parameters are the same as Fig.3. The eigenvalues of matrix $P$ are $0.0003 \pm 2.0494 i, 0.0001 \pm 1.8708 i, 0.0002 \pm 1.5492 i,-0.0001 \pm 2.3452 i$, respectively. Based on Theorem 3 , system (34) generates an oscillatory solution (see Fig.4). 
Especially, when $b_{i}=b(i=2,4,6, \ldots, 2 n), \omega_{i}=\omega, \beta_{i}=\beta, \varepsilon_{i}=\varepsilon, \tau_{i}=\tau(i=1,3,5, \ldots, 2 n-1)$, the synchronization phenomenon appeared. For example, in system (34), we select $b_{i}=-0.00025(i=2,4,6,8), \omega_{i}=0.12, \beta_{i}=0.2, \varepsilon_{i}=$ $0.0005, \tau_{i}=12(i=1,3,5,7)$, the eigenvalues of matrix $P$ are $0.0001 \pm 0.3464 i, 0.0001 \pm 0.3464 i, 0.0001 \pm 0.3464 i, 0.0001 \pm$ $0.3464 i$, respectively. $x_{1}(t)=x_{3}(t)=x_{5}(t)=x_{7}(t), x_{2}(t)=x_{4}(t)=x_{6}(t)=x_{8}(t)$ (see Fig.5 and Fig.6). It was pointed out that for this time delay system, the transient chaos phenomenon does not occur. It may be the difference between a system with time delay and a system without time delay.

\section{Conclusion}

In this paper, we have discussed the dynamical behavior of a $n$ coupled Hamiltonian Duffing equation with time delays. The existence of permanent oscillations which is easy to check, as compared to the bifurcating method that has been proposed in the literature. Some simulations are provided to indicate the effectness of the criterion. The very important transient chaos phenomena do not appear in our simulation.
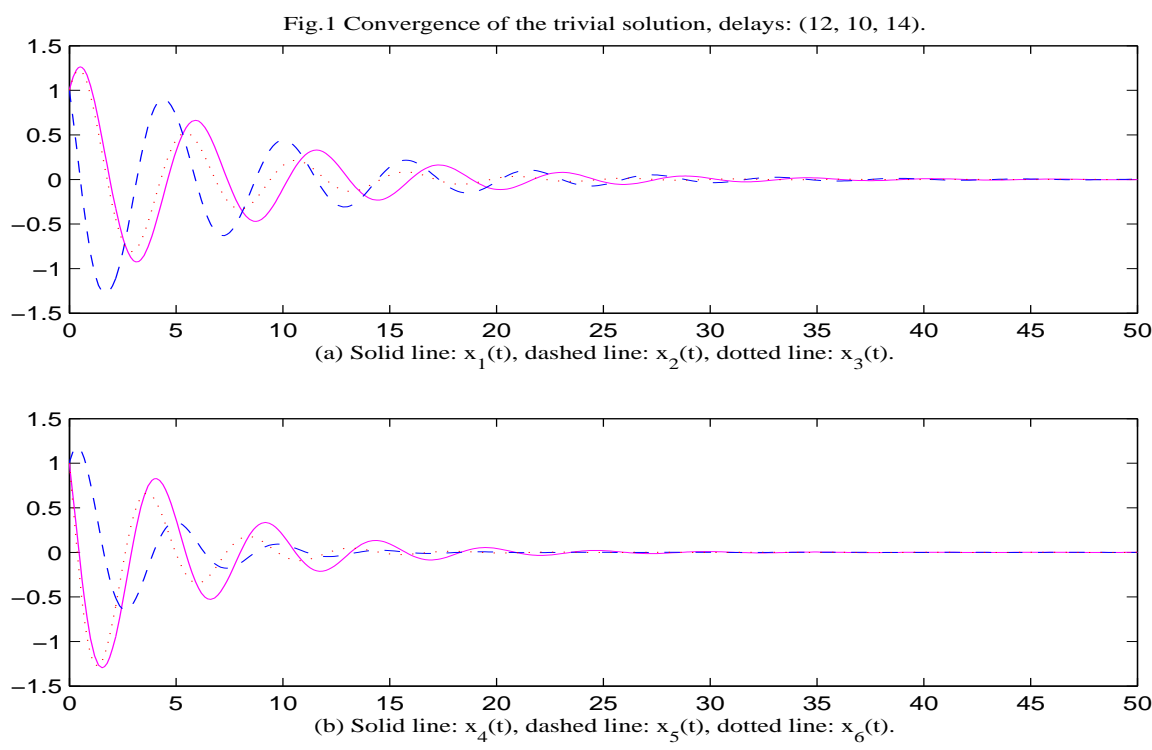

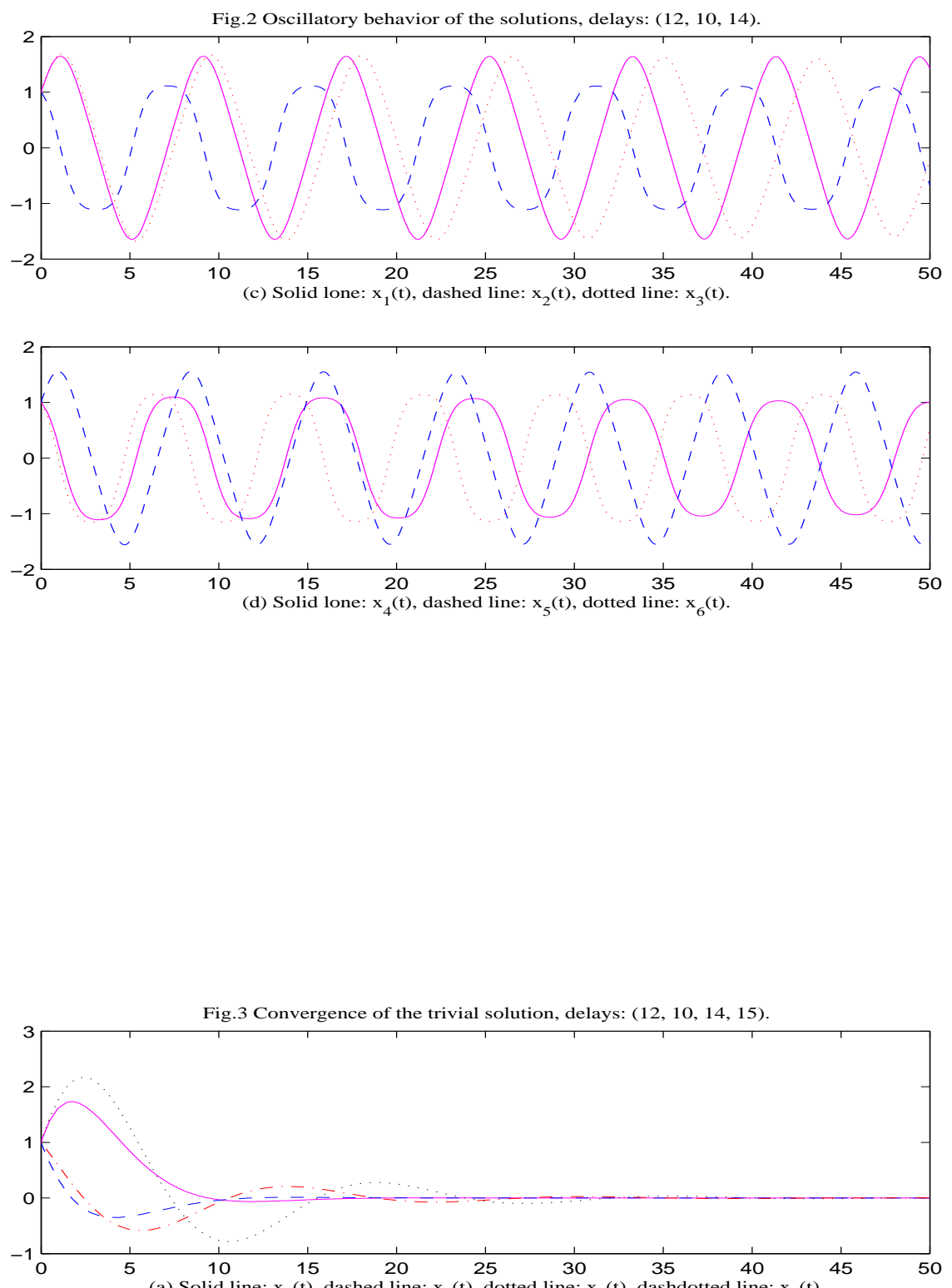

(a) Solid line: $x_{1}(t)$, dashed line: $x_{2}(t)$, dotted line: $x_{3}(t)$, dashdotted line: $x_{4}(t)$.

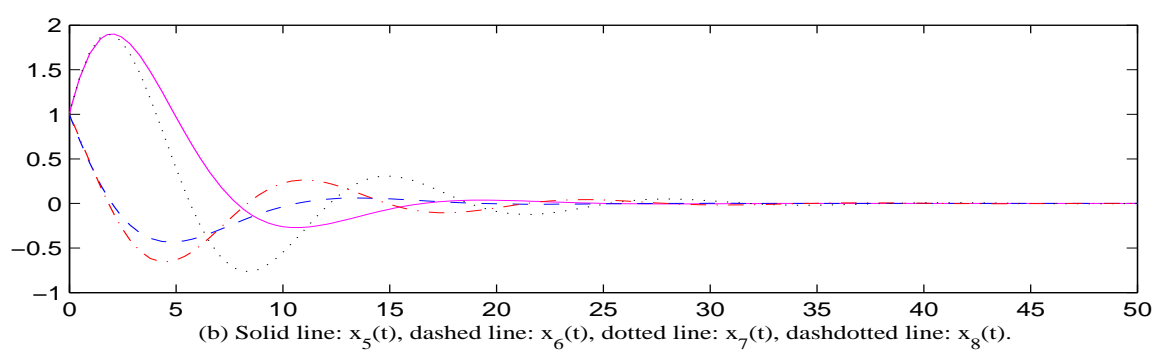



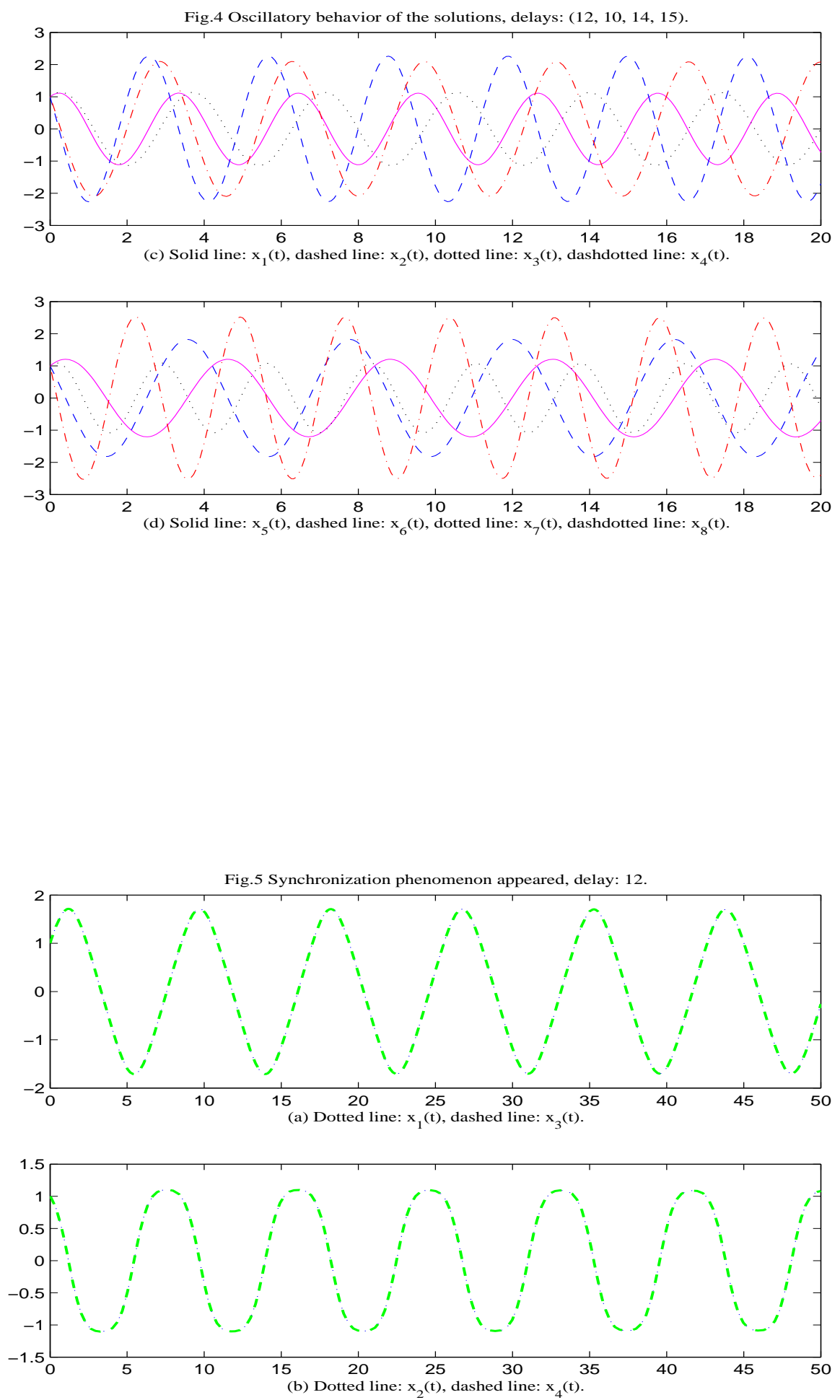

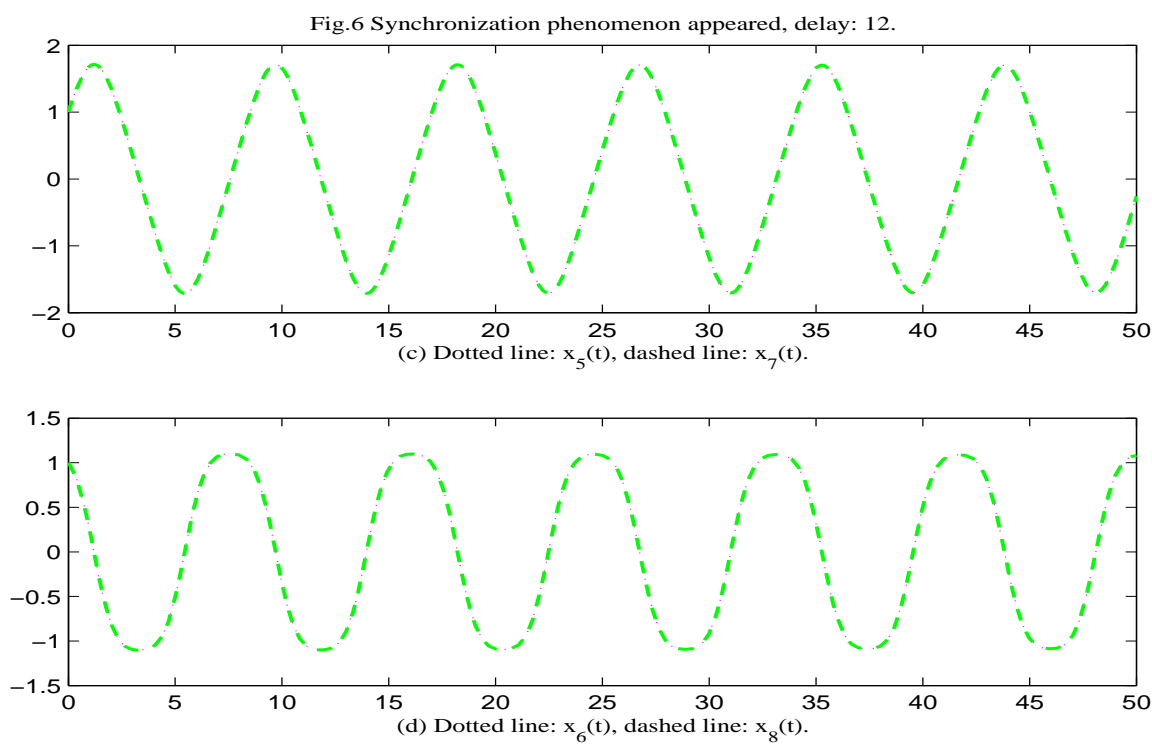

\section{References}

Akhmet, M. U., \& Fen, M. O. (2012). Chaotic period-doubling and OGY control for the forced Duffing equation. Commun Nonlinear Sci Numer Simulat., 17(4), 1929-1946. https://doi.org/10.1016/j.cnsns.2011.09.016

Brzeski, P. Perlikowski, P., \& Kapitaniak, T. (2014). Numerical optimization of tuned mass absorbers attached to strongly nonlinear Duffing oscillator. Commun Nonlinear Sci Numer Simulat., 19(1), 298-310. https://doi.org/10.1016/j.cnsns.2013.06.001

Camargo, J. M., banez, C. A., Guerra, R. M., \& Moctezuma, R. G. (2004). On the parameters identification of the Duffing's system by means of a reduced order observer. Physics Letters A, 331(5), 316-324. https://doi.org/10.1016/j.physleta.2004.09.005

Desoer, C. A., \& Vidyasagar, M. (1977). Feedback System: Input-Output properites. Academic Press, New York.

Hirano, N., \& Rybicki, S. (2003). Existence of limit cycles for coupled van der Pol equations. J. Diff Eqs., 195(1), 194-209. https://doi.org/10.1016/S0022-0396(03)00212-2

Kreindler, E., \& Jameson, A. (1972). Conditions for nonnegativeness of partitioned matrices. IEEE Trans. Auto. Control.,17(1), 147-148. https://doi.org/10.1109/TAC.1972.1099894

Leung, A. Y., Yang, H. X., \& Zhu, P. (2014). Periodic bifurcation of Duffing-van der Pol oscillators having fractional derivatives and time delay. Commun Nonlinear Sci Numer Simulat., 19(4), 1142-1155. https://doi.org/10.1016/j.cnsns.2013.08.020

Mickens, R. E. (1996). Oscillations in Planar Dynamic Systems, World Scientific, Singapore.

Nayfeh, A., \& Mook, D. (1979). Nonlinear Oscillations, Wiley, New York.

Niu, J. C., Shen, Y. J., Yang, S. P., \& Li, S. J. (2017). Analysis of Duffing oscillator with time-delayed fractional-order PID controller. J. Non-Linear Mechanics, 92, 66-75. https://doi.org/10.1016/j.ijnonlinmec.2017.03.014

Pisarchik, A., Reategui, R. J., Salazar, V. J., Lopez, G. J., \& Boccaletti, S. Synchronization of chaotic systems with coexisting attractors. Phys Rev Lett., 96(24), 244102. doi:10.1103/PhysRevlett.96.244102

Qian, Y. H., \& Chen, S. M. Accurate approximate analytical solutions for multi-degree-of-freedom coupled van der PolDuffing oscillators by homotopy analysis method. Commun Nonlinear Sci Numer Simulat., 15(10), 3113-3130.

Rompala, K., Rand, R., \& Howland, H. (2007). Dynamics of three coupled van der Pol oscillators with application to circadian rhythms. Commun Nonlinear Sci Numer Simulat., 12(5), 794-803. https://doi:10.1016/j.cnsns.2005.08.002

Rusinek, R., Weremczuk, A., Kecik, K., \& Warminski, J. (2014). Dynamics of a time delayed Duffing oscillator. Inter. J. 
Non-Linear Mechanics, 65, 98-106. https://doi.org/10.1016/j.jijnonlinmec.2014.04.012

Sabarathinam, S., \& Thamilmaran, K. (2015). Transient chaos in a globally coupled system of nearly conservative Hamiltonian Duffing oscillators. Chaos, Solitons and Fractals, 73, 129-140. https://doi.org/10.1016/j.chaos.2015.01.004

Siewe, M., Tchawoua, C., \& Rajasekar, S. (2012). Parametric resonance in the Rayleigh-Duffing oscillator with timedelayed feedback. Commun Nonlinear Sci Numer Simulat., 17(11), 4485-4493. https://doi.org/10.1016/j.cnsns.2012.02.030

Wang, W. Y., \& Chen, L. J. (2015). Weak and non-resonant double Hopf bifurcations in m coupled van der Pol oscillators with delay coupling. Appl. Math. Model., 39(10-11), 3094-3102. https://doi.org/10.1016/j.apm.2014.11.021

Wen, S. F., Shen, Y. J., Yang, S. P., \& Wang, J. (2017). Dynamical response of Mathieu-Duffing oscillator with fractionalorder delayed feedback. Chaos, Solitons and Fractals, 94, 54-62. https://doi.org/10.1016/j.chaos.2016.11.008

Yalim, J., Welfert, B. D., \& Lopez, J. M. (2017). Evaluation of closure strategies for a periodically-forced Duffing oscillator with slowly modulated frequency subject to Gaussian white noise. Commun Nonlinear Sci Numer Simulat., 44, 144-158. https://doi.org/10.1016/j.cnsns.2016.08.003

Yang, J. H., \& Zhu, H. (2013). Bifurcation and resonance induced by fractional-order damping and time delay feedback in a Duffing system. Commun Nonlinear Sci Numer Simulat., 18(5), 1316-1326. https://doi.org/10.1016/j.cnsns.2012.09.023

Zhang, C. M., Li, W. X., \& Wang, K. Boundedness for network of stochastic coupled van der Pol oscillators with timevarying delayed coupling. Appl. Math. Model., 37(7), 5394-5405. https://doi.org/10.1016/j.apm.2012.10.032

Zhang, J. N., \& Gu, X. S. (2010). Stability and bifurcation analysis in the delay-coupled van der Pol oscillators. Appl. Math. Model., 34(9), 2291-2299. https://doi.org/10.1016/j.apm.2009.10.037

\section{Copyrights}

Copyright for this article is retained by the author(s), with first publication rights granted to the journal.

This is an open-access article distributed under the terms and conditions of the Creative Commons Attribution license (http://creativecommons.org/licenses/by/4.0/). 